\title{
Sonografisch gesteuerte Biopsie fokaler Leberläsionen
}

\author{
Benjamin Meier, Karel Caca, Christoph Klinger
}

Die Leberbiopsie ist eine aussagekräftige Untersuchungsmethode, um unklare diffuse Lebererkrankungen und Rundherde einzuordnen. In den Händen eines erfahrenen Untersuchers ist sie zudem sehr risikoarm. Erfahren Sie hier, wie eine Leberbiopsie fokaler Läsionen Schritt für Schritt abläuft.

\section{Bevor es losgeht}

Indikationen | Die sonografisch gesteuerte Biopsie ist ein wichtiges diagnostisches Instrument, um unklare Leberrundherde histologisch zu beurteilen. Für eine Leberbiopsie sollte man sich nur entscheiden, wenn sich aus ihr therapeutische Konsequenzen ergeben. Keine Biopsie ist erforderlich bei:

- Verdacht auf ein hepatozelluläres Karzinom bei typischer kontrastmittelverstärkter Bildgebung und vorliegender Leberzirrhose

- benignen Leberläsionen mit typischem Kontrastmittelverhalten

- neuen Leberrundherden bei bekanntem metastasierten Tumorleiden

Abb. 1 Punktion in Freihandtechnik in der langen Achse (in-plane). Die Nadel erscheint im sonografischen Bild als echoreiche Linie.

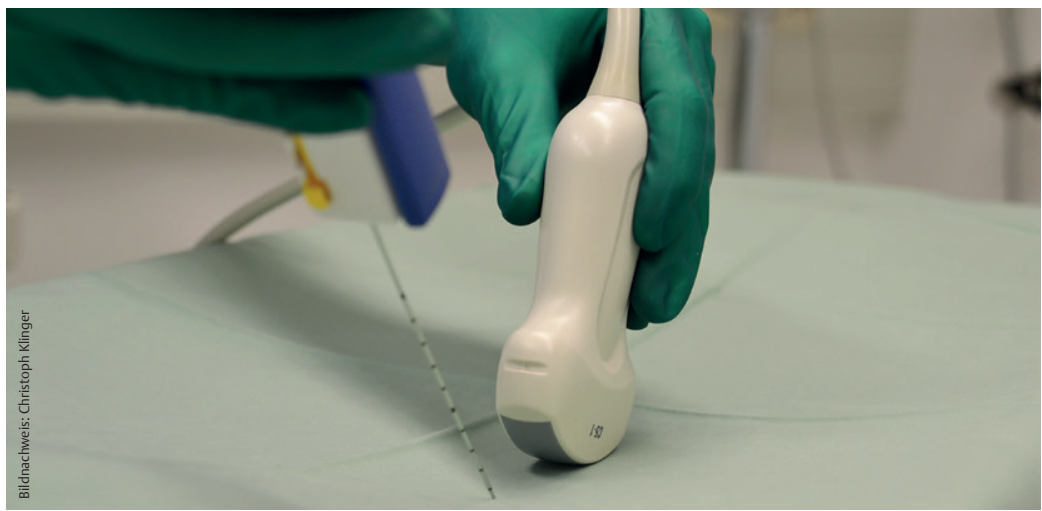

Abb. 2 Punktionsschallkopf (links) und Schallkopf mit Punktionsaufsatz (rechts).

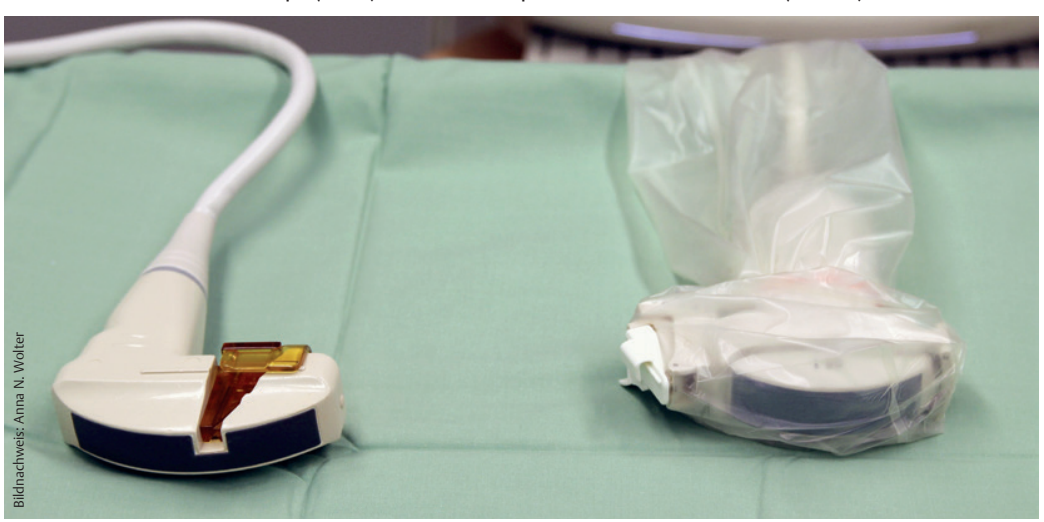

Eine Sondersituation ist die Punktion neuer Leberherde bei bekanntem Tumorleiden, um diese vor einer geplanten zielgerichteten Tumortherapie (z.B. Her2-neu beim Mammakarzinom) immunhistochemisch oder molekulargenetisch zu untersuchen.

Kontraindikationen | Bei der Leberpunktion handelt es sich um einen elektiven Eingriff. Kontraindikationen sind:

- Antikoagulation

- INR $>1,5$ und Thrombozyten $<50000 / \mu l$ (individuelle Blutungsanamnese berücksichtigen)

- Gerinnungsstörungen

- manifester Infekt

- fehlendes Einverständnis

Eine Punktion unter ASS oder NSAR ist möglich, falls eine eindeutige Indikation für die Einnahme besteht [1]. Ansonsten sollte die Einnahme für 5 Tage pausiert werden. Die kombinierte Einnahme von ASS und NSAR sollten Sie aufgrund des erhöhten Blutungsrisikos vermeiden. Antikoagulantien müssen vor dem Eingriff pausiert werden (Heparin / NMH 12 Std., Clopidogrel 5 Tage, neue direkte orale Antikoagulantien 24-48 Std.) [2]. Aszites ist eine relative Kontraindikation. Hier müssen Sie den Patienten über das erhöhte Blutungsrisiko aufklären.

Eine Biopsie fokaler Leberläsionen unter ASS oder NSAR ist möglich.

Nadeltypen und Punktionstechnik | Heutzutage werden überwiegend „Core-biopsy“- oder „TruCut"-Nadeln mit einem Diameter von $18 \mathrm{G}$ in Kombination mit halbautomatischen oder automatischen Biopsiesystemen verwendet. Die Punktion wird sonografisch gesteuert mittels

- Freihandpunktion

- Schallkopfaufsätzen oder

- Punktionsschallköpfen.

Freihandtechnik | Bei der Freihandtechnik sticht man die Punktionsnadel mit 1-2 cm Abstand vom Schallkopf (no-touch-Technik) ein und führt sie frei mit der Hand in der Schallkopfebene (lange Achse=in-plane; ( Abb. 1). Diese Technik ist 


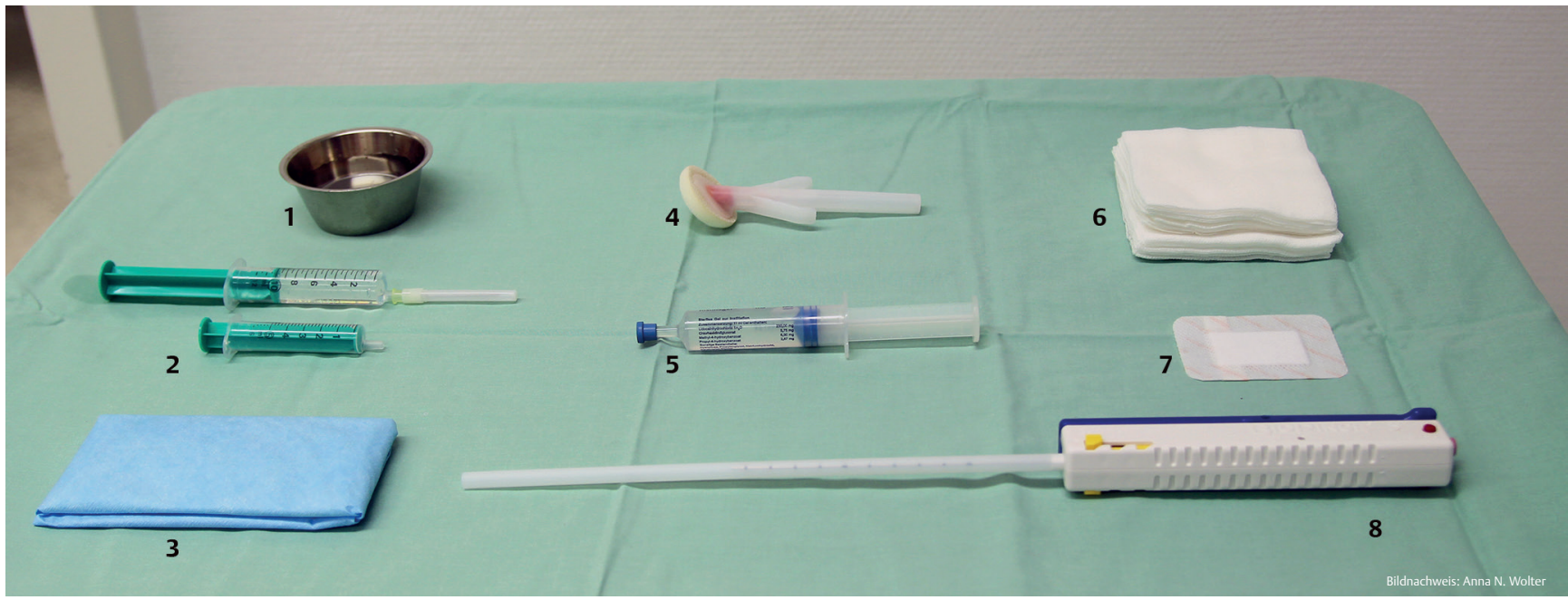

kostengünstig und erlaubt eine große Flexibilität, da sie mit jedem Sonografiegerät möglich ist und die Punktionsrichtung frei gewählt werden kann.

Für die Freihandtechnik ist etwas Übung erforderlich. Unerfahrene Untersucher sollten sich daher zunächst auf große, oberflächlich gelegene Läsionen beschränken.

Punktionshilfen | Bei Schallkopfaufsätzen und Punktionsschallköpfen ( Abb. 2) lassen sich meist nur 2 bzw. 3 verschiedene Punktionswinkel auswählen. Sie können die Punktionsrichtung somit nicht frei wählen. Allerdings erlauben diese Punktionshilfen auch tief gelegene kleine Läsionen in Nachbarschaft kritischer Strukturen (z.B. Blutgefäße, Gallenblase) sicher zu punktieren. Die Punktion mit Punktionshilfen ist jedoch kostenintensiver als die Freihandtechnik.

Komplikationen | Die sonografisch gesteuerte Biopsie fokaler Leberläsionen ist ein relativ risikoarmes Verfahren. Eine klinisch relevante Blutung tritt bei ca. 0,32\% der Patienten auf, wobei stark vaskularisierte und oberflächlich gelegene Läsio-

Abb. 4 Lokalanästhesie mit $10 \mathrm{ml}$ Scandicain $1 \%$.

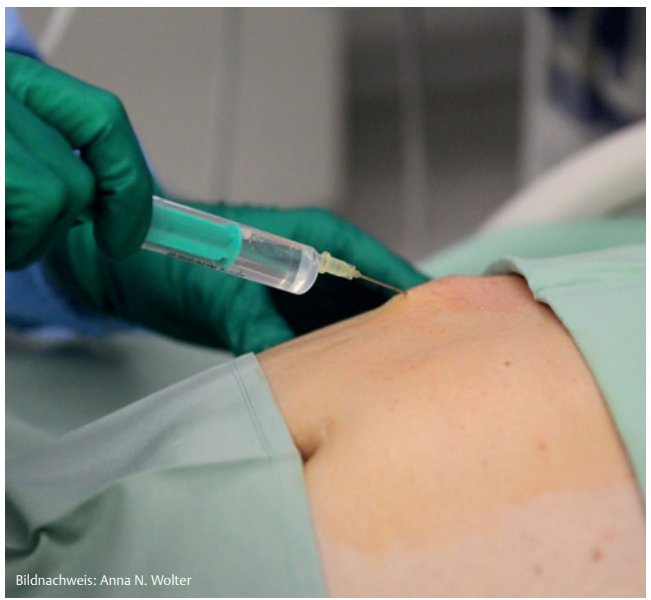

nen (z.B. HCC, neuroendokrine Tumoren, malignes Melanom) ein etwas erhöhtes Risiko haben. Die Letalität von Leberbiopsien liegt bei ca. $0,05 \%$, bei der Punktion fokaler Leberläsionen liegt sie bei ca. 0,09\% [3]. Bei kontinuierlicher sonografischer Darstellung der Punktionsnadel kommt es nur sehr selten zu einer Verletzung von Nachbarorganen. Auch infektiöse Komplikationen sind sehr selten, solange Sie die nötigen Hygienemaßnahmen beachten. Die Häufigkeit einer Stichkanalmetastasierung ist in der Literatur umstritten. [4, 5]

Vorbereitung des Patienten | Der Patient sollte 24 Stunden vor dem Eingriff über die möglichen Komplikationen aufgeklärt werden. Für den Eingriff selbst sollte er für 4-6 Stunden nüchtern sein. Wie er für die Punktion gelagert wird, ist abhängig vom gewählten Punktionsweg.

Letzte Vorbereitungsschritte | Vor dem Eingriff sind folgende Vorbereitungen zu treffen:

- Überprüfung der Indikation und möglicher Kontraindikationen

- Überprüfung der Medikation und der aktuellen Laborparameter

- Anlage einer Venenverweilkanüle

Abb. 5 Sonografische Einstellung der Läsion.

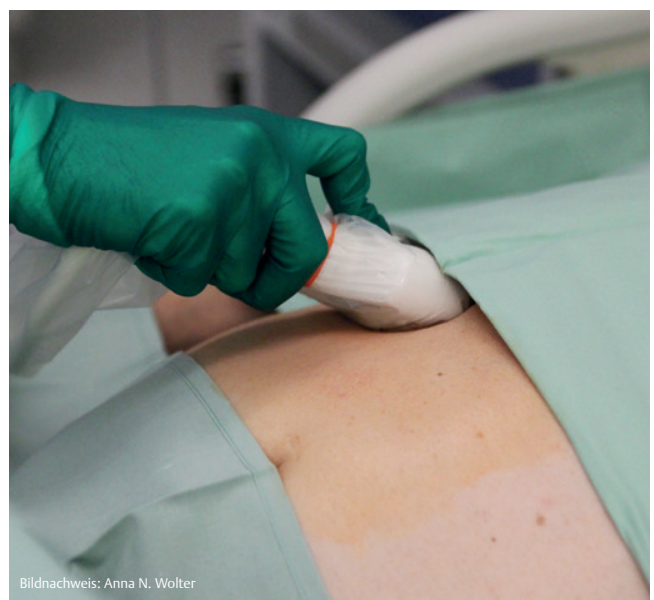

Abb. 3 Materialien für die Leberpunktion. (1) Steriles Gefäß mit NaCl 0,9\%, (2) Spritzen mit Lokalanästhetikum, (3) sterile Schallkopfhülle, (4) Hautdesinfektionsmittel, (5) steriles Sonographie-Gel, (6) sterile Kompressen, (7) Pflaster, (8) Punktionsnadel (18 G Biopince $^{\circledR}$ ). 


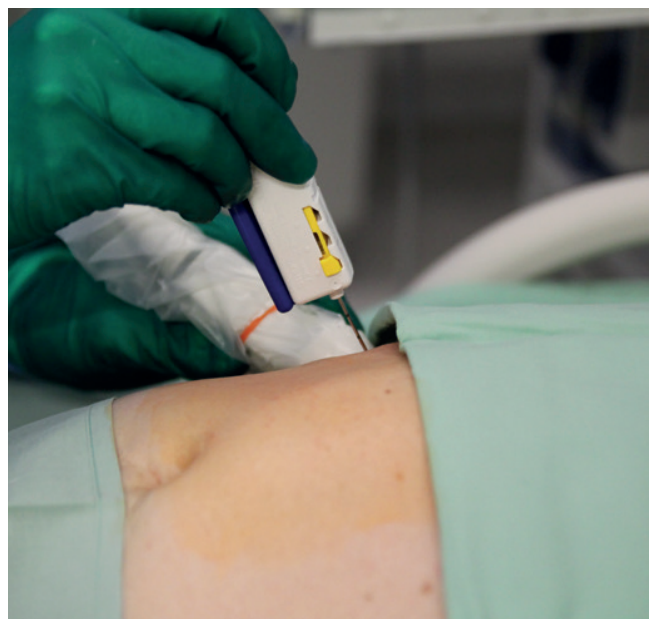

Abb. 6 Die Punktionsnadel wird ca. $1-2 \mathrm{~cm}$ neben dem Schallkopf eingestochen.

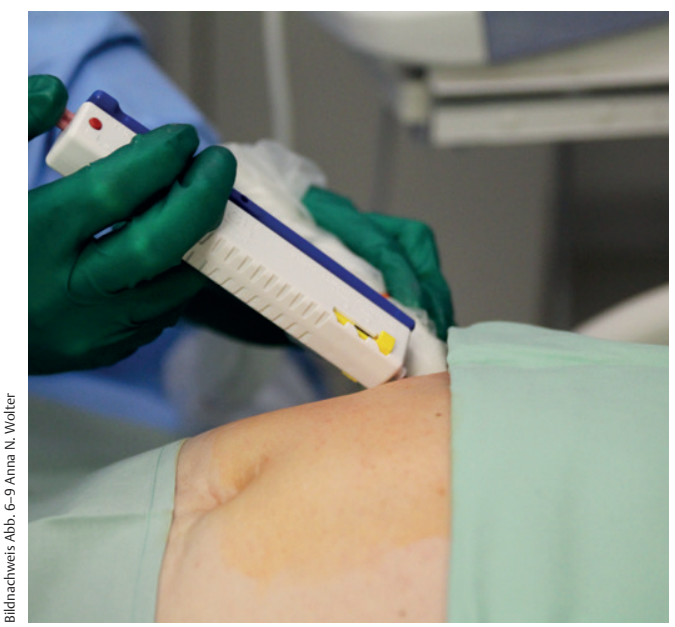

Abb. 8 Der Punktionsmechanismus wird ausgelöst.

- Monitoring (regelmäßige Dokumentation von Blutdruck, Herzfrequenz und Sauerstoffsättigung)

- Vorbereitung des sterilen Tisches $(\bullet$ Abb. 3)

- Sonografische Lokalisation der Läsion

- Festlegung des optimalen Punktionsweges (Gewebsdeckung $>5 \mathrm{~mm}$, ausreichender Abstand zu Gefäßen und Gallenblase)

- Lagerung des Patienten abhängig vom gewählten Punktionsweg

\section{So wird's gemacht: Leberbiopsie}

Hygienemaßnahmen | Um infektiöse Komplikationen zu vermeiden, sollten Sie folgende Punkte beachten:

- Händedesinfektion

- sterile Handschuhe

- Hautdesinfektion

- steriles Abdecktuch

- steriles Schallgel oder alkoholisches Desinfektionsmittel

- Reinigung und Desinfektion des Schallkopfes

- sterile Schallkopfhülle (bei „no-touch“-Technik nicht erforderlich)

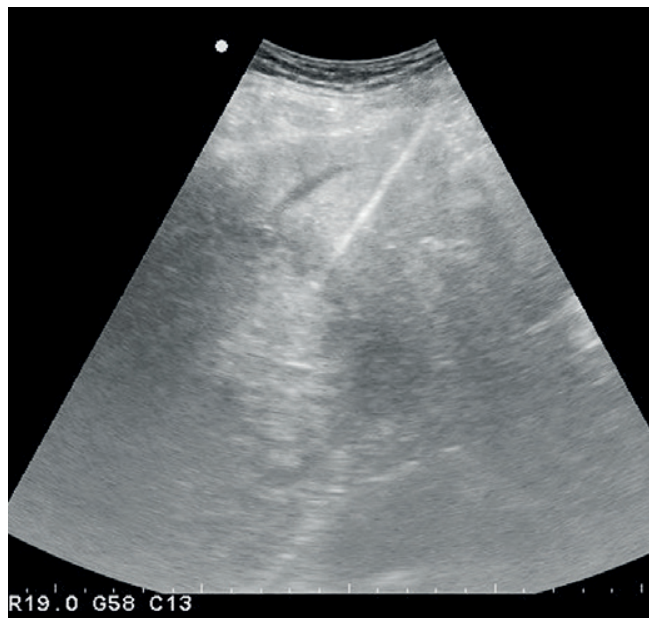

Abb. 7 Sonografische Kontrolle während der Biopsie.

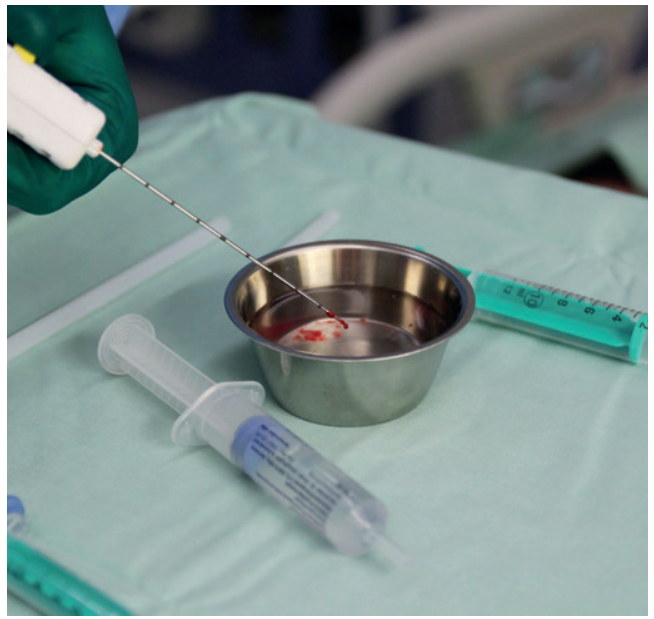

Abb. 9 Die Probe wird in $\mathrm{NaCl}$ 0,9\% gegeben.

Anästhesie | Während der Punktion sollte der Untersucher dem Patienten alle Handlungen ankündigen. Nach sorgfältiger Hautdesinfektion und sterilem Abdecken erfolgt die Lokalanästhesie mit $10 \mathrm{ml}$ Scandicain $1 \%$ ( $\triangleright$ Abb. 4). In Einzelfällen können Sie mit Midazolam sedieren.

Einstellung und Punktion | Die Läsion wird zunächst sonografisch eingestellt ( Abb. 5). Hierbei können vor allem bei kranial gelegenen Läsionen Atemmanöver hilfreich sein. Die Punktionsnadel wird ca. 1-2 cm neben dem Schallkopf eingestochen (,no-touch-Technik“; > Abb. 6). Je nach Punktionsnadel ist vorher eine Stichinzision mit einem Skalpell notwendig. Anschließend wird die Punktionsnadel unter ständiger sonografischer Kontrolle vorgeführt und die Läsion punktiert $(\checkmark$ Abb. 7 und 8 ).

Cave Achten Sie darauf, dass eine ausreichende Gewebedeckung $(>5 \mathrm{~mm}$ ) gegeben ist und keine Gefäße im Punktionsweg liegen. Punktieren Sie möglichst nicht in Richtung Gefäße oder Gallenblase. 


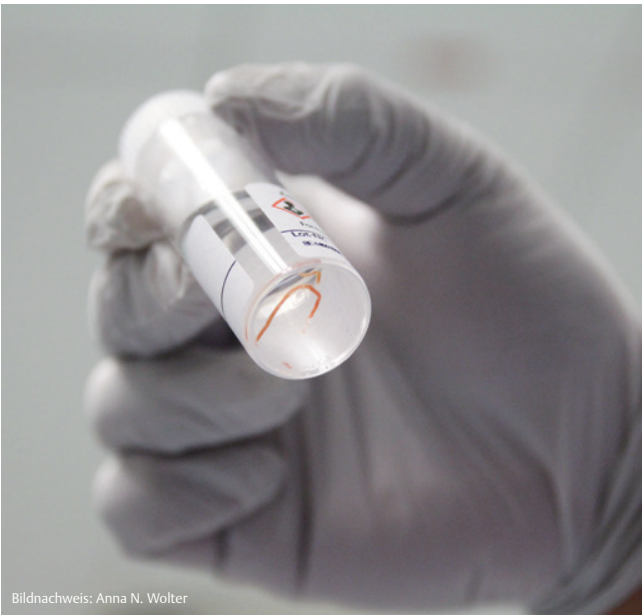

Abb. 10 Die Probe wird in Formalin 4\% umgefüllt und optisch kontrolliert.

Kontrolle des Lebergewebes | Nachdem man die Punktionsnadel entfernt hat, wird der Gewebezylinder ausgelöst und in $\mathrm{NaCl} 0,9 \%$ gegeben ( Abb. 9). Die Nadel bleibt dabei steril und kann ggf. für eine zweite Biopsie verwendet werden. Überführen Sie die Probe in Formalin 4\% und kontrollieren Sie sie optisch $(\mathbf{A b b} . \mathbf{1 0})$.

Normales Lebergewebe hat eine rotbraune Farbe. Pathologische Veränderungen sind hingegen meist weißlich.

Falls Unsicherheit besteht, ob ausreichend gutes Material vorliegt, sollten Sie erneut biopsieren. Bei nekrotisch zerfallenden Läsionen sind immer mindestens zwei Punktionen (zentral und peripher) notwendig, um neben nekrotischem Material auch sicher vitales Gewebe zu erhalten.

\section{Nach dem Eingriff}

Patientenlagerung I Nach der Punktion wird ein steriles Pflaster auf die Punktionsstelle geklebt. Der Patient legt sich auf die Seite, so dass die Punktionsstelle auf einer Kissenrolle oder einem Sandsack zu liegen kommt ( Abb. 11). Hierdurch soll sich das Risiko einer Nachblutung minimieren.

Klinisch beobachten | Bezüglich der Nachsorge gibt es keine allgemeingültigen Empfehlungen. Da sich $80 \%$ der Komplikationen innerhalb der ersten 24 Stunden ereignen, ist eine klinische Beobachtung während dieser Zeit notwendig. Wichtig ist, dass die Nachsorge abteilungsintern standardisiert und dokumentiert wird (Festlegung einer „standard operating procedure“).

Kontrolle I In unserer Klinik soll der Patient nach Punktion eine Bettruhe (Lagerung auf Kissenrolle/Sandsack) für 4 Stunden einhalten. Wäh-

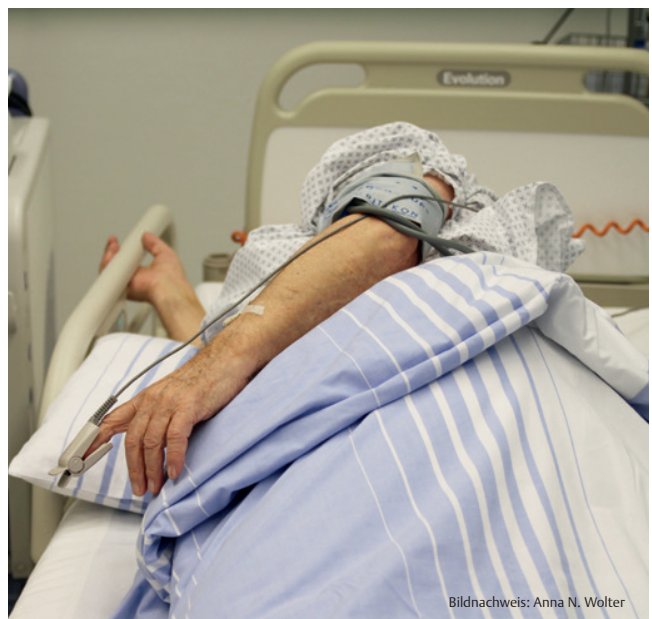

Abb. 11 Lagerung des Patienten auf einer Kissenrolle.

rend dieser Zeit bleibt er nüchtern. Blutdruck und Herzfrequenz werden über 2 Stunden halbstündlich, dann über weitere 2 Stunden stündlich und zuletzt bis 22 Uhr alle 2 Stunden gemessen. Das anschließende Prozedere hängt individuell vom Verlauf ab. Eine Blutbildkontrolle führen wir 4 Stunden nach Punktion und am Folgetag durch. Eine sonografische Kontrolle ist routinemäßig nicht erforderlich, sollte jedoch erfolgen bei:

- Beschwerden (abdominelle Schmerzen, auffällige Vitalparameter, Abfall des Hämoglobins) nach dem Eingriff

- Hochrisikopatienten (z.B. eingeschränkte Gerinnungsfunktion, hypervaskularisierte Läsion, schwierige Punktion, gefäßnahe/kapselnahe Lage der Läsion)

\section{Wichtiges in Kürze}

- Die sonografisch gesteuerte Biopsie der Leber ermöglicht durch die gezielte Punktion eines Leberherdes dessen histologische Beurteilung.

- Eine Punktion sollte nur erfolgen, wenn sich hieraus therapeutische Konsequenzen ergeben.

- Bei Beachtung der Kontraindikationen ist die Komplikationsrate beim erfahrenen Untersucher gering.

- Die postinterventionelle Überwachung sollte stets nach abteilungsinternen Standards (SOP) erfolgen und dokumentiert werden.

- Eine routinemäßige sonografische Kontrolle ist nur bei Hochrisikopatienten erforderlich. Bei Beschwerden ist sie umgehend durchzuführen.

\section{Literatur}

1 Atwell TD, Smith RL, Hesley GK et al. Incidence of bleeding after 15181 percutaneous biopsies and the role of aspirin. Am J Roentgenol 2010; 194: 784-789

2 Patel IJ, Davidson JC, Nikolic B et al. Consensus guidelines for periprocedural management of coagulation status and hemostasis risk in percutaneous image-guided interventions. J Vasc Interv Radiol 2012; 23: 727-736

Vollständiges Literaturverzeichnis unter http://dx.doi.org/10.1055/s-0041-102520

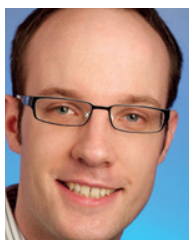

Dr. med. Benjamin Meier ist Assistenzarzt der Klinik für Innere Medizin, Gastroenterologie, Hämato-Onkologie, Diabetologie und Infektiologie am Klinikum Ludwigsburg. benjamin.meier@kliniken-lb.de

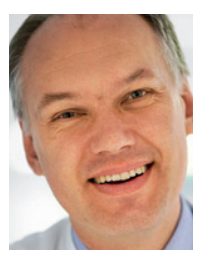

Prof. Dr. med. Karel Caca ist Ärztlicher Direktor der Klinik für Innere Medizin, Gastroenterologie, Hämato-Onkologie, Diabetologie und Infektiologie am Klinikum Ludwigsburg. karel.caca@kliniken-lb.de

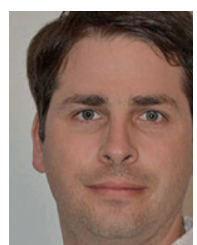

Dr. med. Christoph Klinger ist Oberarzt der Klinik für Innere Medizin, Gastroenterologie, Hämato-Onkologie, Diabetologie und Infektiologie am Klinikum Ludwigsburg und Leiter der Sonographie. christoph.klinger@kliniken-lb.de

Interessenkonflikt

Die Autoren geben an, dass kein Interessenkonflikt besteht

DOI 10.1055/s-0041-102520 Dtsch Med Wochenschr 2015; 140: $820-823$

(c) Georg Thieme Verlag KG . Stuttgart · New York .

ISSN 0012-0472 
3 Strobel D, Bernatik T, Blank W et al. Incidence of Bleeding in 8172 Percutaneous Ultrasound-Guided Intraabdominal Diagnostic and Therapeutic Interventions - Results of the Prospective Multicenter DEGUM-Interventional Ultrasound Study (PIUS Study). Ultraschall in Med 2015; 36: 122-131

4 Silva MA, Hegab B, Hyde C et al. Needle track seeding following biopsy of liver lesions in the diagnosis of hepatocellular cancer: a systematic review and meta-analysis. Gut 2008; 57: 1592-1596

5 Robertson EG, Baxter G. Tumour seeding following percutaneous needle biopsy: The real story! Clinical Radiology 2011; 66: 1007-1014 